\title{
Surfaces
}

\section{Politiques de l'autorité. Le pouvoir de celui qui « n’est rien »}

\section{Jean-Ernest Joos}

Volume 6, 1996

LES ÉCONOMIES DISCURSIVES DU SAVOIR ET DE LA CULTURE DANS LE SILLAGE DE L'OEUVRE DE BILL READINGS

THE DISCURSIVE ECONOMIES OF KNOWLEDGE AND CULTURE, WITH CONSTANT REFERENCE TO THE WORK OF BILL READINGS

URI : https://id.erudit.org/iderudit/1064851ar

DOI : https://doi.org/10.7202/1064851ar

Aller au sommaire du numéro

\section{Éditeur(s)}

Les Presses de l’Université de Montréal

ISSN

1188-2492 (imprimé)

1200-5320 (numérique)

Découvrir la revue

Citer cet article

Joos, J.-E. (1996). Politiques de l'autorité. Le pouvoir de celui qui « n'est rien ». Surfaces, 6. https://doi.org/10.7202/1064851ar
Résumé de l'article

Ce texte se propose de mettre en évidence une conception de l'autorité qui traverse la modernité et la postmodernité, celle d'une autorité qui se légitime par son invisibilité. Le concept d'autorité désigne ici, non seulement le droit de parole, mais aussi le lieu où l'institution prend corps. Il s'agit donc d'analyser les conséquences politiques de cette forme d'incorporation institutionnelle qui a été aussi celle de l'université, notamment dans la constitution de la figure du " maître » et des frontières disciplinaires. Il me semble clair en effet que c'est cette forme d'autorité qui est remise en question par l'introduction des politiques du corps (Gays, Lesbiennes, Queer, Afro-américaines...) dans cette configuration institutionnelle fondée sur le pouvoir de l'invisibilité.
Copyright ( Jean-Ernest Joos, 1996

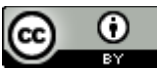

Ce document est protégé par la loi sur le droit d'auteur. L’utilisation des services d’Érudit (y compris la reproduction) est assujettie à sa politique d'utilisation que vous pouvez consulter en ligne.

https://apropos.erudit.org/fr/usagers/politique-dutilisation/ 


\section{Politiques de l'autorité. Le pouvoir de celui qui n'est rien}

Jean-Ernest Joos

k26731@er.uqam.ca

Surfaces Vol. VI.206 (v.1.0F - 15/10/1996) - ISSN:

1188-2492

Tout texte reste la propriété de son auteur. Néanmoins, Surfaces demande d'être citée à l'occasion de toute autre publication du texte en question.

\section{RÉSUMÉ}

Ce texte se propose de mettre en évidence une conception de l'autorité qui traverse la modernité et la postmodernité, celle d'une autorité qui se légitime par son invisibilité. Le concept d'autorité désigne ici, non seulement le droit de parole, mais aussi le lieu où l'institution prend corps. Il s'agit donc d'analyser les conséquences politiques de cette forme d'incorporation institutionnelle qui a été aussi celle de l'université, notamment dans la constitution de la figure du maître et des frontières disciplinaires. Il me semble clair en effet que c'est cette forme d'autorité qui est remise en question par l'introduction des politiques du corps (Gays, Lesbiennes, Queer, Afro-américaines...) dans cette configuration institutionnelle fondée sur le pouvoir de l'invisibilité.

\section{ABSTRACT}

This text aims to delineate a conception of authority that runs through modernity and postmodernity and whose legitimacy rests on its invisibility. Here the concept of authority 
means not only the right to speak but also the place where the institution takes form. It is therefore a question of analyzing the political consequences of the form of institutional incorporation that includes also the university, specifically in its constitution of the figure of the master and disciplinary boundaries. In effect, in this institutional configuration founded on the power of invisibility, it seems clear that introducing (gay, lesbian, queer, African-American) body politics puts into question precisely this form of authority.

Rien n'est moins évident que de repérer où l'autorité $a$ lieu dans le corps institutionnel. On peut certes reconnaître dans le corps du maître (professeur, enseignant, conférencier) la médiation par laquelle passe et le savoir et la juridiction institutionnelle. Cependant, comment savoir ce qui dans ce corps présent appartient véritablement au corps institutionnel et ce qui relève de la contingence et du privé? Faut-il savoir, peut-on savoir? La question est d'autant plus tordue que souvent l'autorité professorale se manifeste précisément dans cette zone non-réglementée par l'institution qui recouvre toutes les décisions particulières à prendre aussi bien dans l'évaluation que dans le choix du contenu des cours. La pensée politique de Lyotard nous a appris à reconnaître dans le moment du jugement une des formes politiques les plus décisives et en même temps la plus difficile à identifier. Tel enseignant introduit plus de contenu spécifique (sur les femmes, les Gais, ou les questions raciales) que tel autre dans des cours pourtant identiques dans leurs définitions. Bien plus, le corps de l'autorité ne se réduit pas au corps de l'enseignant, mais englobe tous les effets que sa présence produit dans le corps collectif des récepteurs. Les modalités de leur droit de parole sont déterminées par la présence physique de ce tiers qui détient le droit de parler en premier. Or les effets de l'autorité sont souvent invisibles. Que l'on songe simplement à tous les contrecoups du transfert ou de la séduction, à toutes les frontières et hiérarchies, notamment entre les sexes et les identités sexuelles, ou culturelles, qui structurent la société civile et qui se reflètent inéluctablement dans les rapports au sein de l'institution.

A toutes ces questions essentielles, je voudrais joindre une analyse de la politique institutionnelle de l'autorité. J'entends par là le mode par lequel l'autorité acquiert sa 
légitimité, non pas sous le couvert de la fonction occupée, mais dans sa singularité corporelle. Il y a en effet un procès de légitimation bien déterminé qui permet à un corps-médiateur de s'élever au statut de corps institutionnel . Un tel procès de légitimation n'est pas visible pour la simple raison qu'il repose justement sur l'invisibilité comme octroi du droit de parole. Les effets d'une telle légitimation sont eux aussi invisibles, mais bien réels, dans la mesure où l'invisibilité de la figure de l'autorité n'est que le mode par lequel l'autorité s'inscrit dans des interactions effectives. La figure du maître invisible, repérable, dès la fondation kantienne de l'université, est en fait bien plus ancienne dans la pensée politique moderne, et participe de ce que j'appellerai une éthique de l'invisibilité. Et la politique que cette éthique fonde est précisément ce qui est le plus contesté et même le plus menacé par les nouvelles politisations du savoir qu'impliquent les Études à caractères spécifiques, telles que les Études féministes, les Études sur la femme, les Études gaies, lesbiennes, Queer, les Études afroaméricaines, et liées à des cultures vécues. Ces Études, en déplaçant la frontière du privé et du public, entendent révéler le corps de l'autorité comme enjeu et lieu politiques, refusant de le réduire à une simple médiation. Du coup, c'est aussi le projet moderne des Lumières, celui d'une accessibilité libre et illimitée de tous au savoir, qui se trouve questionné, la légitimité de l'autorité invisible reposant très exactement sur le rêve d'un médiateur dont la spécificité corporelle ne serait jamais un obstacle à sa circulation dans toutes les sphères du savoir.

\section{La question de l'autorité}

La difficulté de toute réflexion sur l'autorité tient à ce que l'autorité est toujours à la fois la présence de celui qui est là et son absence à soi qui résulte de ce que la légitimité n'est jamais tout à fait immanente et qu'elle est donnée . Cette donation ne relève pas de la désignation par l'institution, qui à l'inverse s'inscrit à l'intérieur de celle-là. C'est pourquoi, il faut procéder en deux temps: repérer comment la légitimité se donne, puis les effets politiques de la différence entre le don de la légitimité et le corps qui le reçoit. Il n'est pas sûr en effet que l'éthique de l'invisibilité soit contestable en tant que procès de légitimation; la question sera peut-être de savoir s'il est politiquement acceptable de réduire la question de l'autorité à sa légitimation. Bref, il faudra se demander ce que la fondation institutionnelle laisse de côté. 
L'éthique de l'invisibilité accorde des droits sur le fond de devoirs. Je désigne par cette appellation à la fois le geste théorique et politique par lequel la Modernité a ramené la question de l'autorité à celle de l'origine du droit et le discours fondateur qui s'est constitué comme solution incontournable à la question ainsi posée. Cette circularité est très bien repérée par Derrida dans Force de loi, bien que dans un langage conceptuel différent, en référence aux pensées de Montaigne et de Pascal. Derrida cite Pascal:

La coutume fait toute l'équité, par la seule raison qu'elle est reçue; c'est le fondement mystique de son autorité. Qui la ramène à son principe, l'anéantit (29).

C'est la donation de l'autorité qui fait l'autorité, la révélation de la donation en tant que telle signifie la destruction de l'autorité. On voit ici poindre ce que je nomme l'éthique de l'invisibilité, dans la mesure où pour Pascal et Montaigne, il n'y a de justice que si le fondement de l'autorité demeure invisible. Cette invisibilité n'est jamais purement factuelle, elle est le résultat attendu de notre respect de l'autorité. Et le paradoxe fondateur de la lecture derridienne est qu'elle s'appuie sur cette même invisibilité pour déconstruire le fondement de l'autorité et faire valoir cette déconstruction comme la justice même. Le retrait de l'origine, son caractère mystique, fonde l'autorité, la déconstruction identifie ce retrait comme le moment indécidable de la fondation où se décide toute justice. En ce sens, l'éthique de l'invisibilité se retrouve plus dans la pensée derridienne que dans celles de Pascal et de Montaigne, dans la mesure où elle est ici le fondement en retrait et là la loi, le il faut de toute décision juste en ce que la justice de la décision même est toujours à venir (57). Cependant, il n'est pas dans mon intention d'entrer dans un débat avec Derrida, puisque ce n'est pas la structure et la justification de l'éthique de l'invisibilité qui m'intéresse ici, mais la forme qu'elle prend et les effets politiques et institutionnels qu'elle produit. C'est pourquoi, je me tourne maintenant plutôt vers Kant, et vers ce qui s'y apparente dans la pensée politique de Rousseau.

Dans le Contrat social, de Rousseau, il y a une entrée sur le législateur (Du législateur). Là Rousseau cherche à décider quelle intelligence est la plus apte à écrire les lois, bref qui doit être l'élu. La question du législateur est d'emblée paradoxale, puisqu'il est l'énonciateur des 
lois sans pouvoir prétendre pourtant aucunement à l'autorité qui revient à la seule volonté générale.

Ainsi l'on trouve à la fois dans l'ouvrage de la législation deux choses qui semblent

incompatibles: une entreprise au-dessus de la force humaine et, pour l'exécuter, une autorité qui n'est rien.

Il faut bien voir que ce paradoxe n'aurait pas lieu si la question de l'autorité n'avait pas été réduite à celle de l'élection. Et je songe bien entendu à la formation même de la volonté générale sur la base d'un contrat social égalitaire. C'est parce que l'on constitue un espace égalitaire que le lieu de l'autorité est désigné comme l'exception qui doit se démarquer du tout. Ce caractère extraordinaire du législateur se traduit alors comme une étonnante compétence temporelle. Dans la mesure ou l'effacement de sa particularité est un trait qu'il partage avec tous les membres de la volonté générale, il n'aura en propre que le pouvoir de le faire maintenant, bref il est celui qui peut travailler dans un siècle et jouir dans un autre. Et le respect de ses lois exige que les hommes fussent avant les lois ce qu'ils doivent devenir par elles . Du coup, Rousseau propose l'instrumentalisation de la religion. Il faut que le législateur mette ses lois dans la bouche des immortels pour entraîner par l'autorité divine ceux que ne pourrait ébranler la prudence humaine . Cependant, cette religion du faire-semblant ne doit pas nous détourner de la reconnaissance du devoir sublime qui l'impose comme devoir. Il y a à tout cela un fondement éthique qui élit le législateur comme être exceptionnel au prix d'un devoir, celui de n'être rien que médiation et de créer les conditions de la croyance. Et ce fondement éthique est la temporalité même (le temps du jugement) de la volonté générale, l'écart entre la volonté du peuple et son jugement. De lui-même, le peuple veut toujours le bien, mais de lui-même, il ne le voit pas toujours (De la loi). Il resterait bien sûr à analyser la forme complexe que prend la dimension de l'éthique dans une pensée qui prétend, dès son entrée en matière, prendre les hommes tels qu'ils sont. Pourtant, on peut déjà conclure sur ce qui rapproche Rousseau de Montaigne et de Pascal : cet artifice d'une autorité qui ne vaut qu'en tant que le retrait de son origine est respectée, aussi bien par l'autorité elle-même que par les sujets. Dans le projet rousseauiste, ce retrait se lit en ce que le législateur n'est proprement rien, rien en particulier, il n'est que l'avenir du peuple. Ce retrait n'est pas une chose réelle, il n'existe que comme principe d'élection pour déterminer celui qui a une raison sublime, capable 
d'effacement, et pour imposer au peuple la croyance en l'origine divine de cette désignation. Ainsi, se fonde l'État.

\section{L'éthique de l'invisibilité}

Le concept d'une éthique de l'invisibilité n'apparaît pourtant explicitement que dans la pensée kantienne. Non pas que l'invisibilité comme principe de l'autorité soit proprement kantienne, mais la fondation éthique de ce principe s'inscrit enfin comme élément structurel d'une pensée politique. 1784, avec le texte sur les Lumières, le concept d'autorité reçoit la marque historique de l'Aufklärung. L'autorité est donnée à tous, à la condition de faire un bon usage de sa raison, un usage public à travers des écrits circulant et constituant la société universelle des savants. Il s'agit bien d'une position sur l'autorité, sur le droit de parler publiquement, qui fait référence explicitement à la donation de l'autorité. Il y est reconnu à chacun le droit de jouir d'une liberté sans bornes d'utiliser sa propre raison et de parler en son nom propre (50). La phrase dans son balancement assigne la raison à résidence dans la parole singulière. L'usage public de la raison est le lieu où l'on s'autorise de soi-même. 1785, avec les Fondements de la métaphysique des moeurs, dans les formulations même de l'impératif catégorique qui fonde l'autonomie de la raison, surgissent les premières traces de l'éthique de l'invisibilité. Une fois reconnu qu'une volonté bonne est celle qui doit se regarder comme instituant une législation universelle dans le règne des fins, Kant peut préciser la nature de cette législation, par une des nombreuses formules de l'impératif moral.

Tout être raisonnable doit agir comme s'il était toujours par ses maximes un membre législateur du règne universel des fins (166).

La notion de membre législateur est précisée quelques pages plus tôt par la distinction entre un membre et un chef dans le règne des fins (158). Le membre est celui qui, tout en donnant des lois universelles, n'en est pas moins soumis à ces lois. Le chef est celui qui n'est soumis à aucune volonté étrangère. Il en résulte bien sûr que seul Dieu peut être chef. La structure politique est alors claire, elle est triangulaire avec Dieu en position de tiers, c'est-à-dire occupant le lieu de l'origine de la législation. Son originalité pourtant n'est évidemment pas là. Elle est dans la notion même de membre 
législateur qui permet à un être fini soumis au devoir d'occuper la place du tiers tout en se voyant refuser toute exclusivité en ce lieu. La distinction entre chef et membre sert alors à identifier le lieu de la législation et Dieu à en marquer l'unicité. Chaque membre législateur est ainsi législateur d'une même législation et Dieu luimême n'a aucune autre exclusivité que celle de ne pas avoir à être membre. D'une part, on a un Dieu dont l'unicité ne réside pas dans sa loi, mais seulement dans sa capacité à exclure les êtres raisonnables finis de la place de chef. D'autre part, on a des êtres finis qui occupent un lieu universel de législation qu'ils ne peuvent jamais s'approprier. Être membre, c'est structurellement être un membre parmi d'autres. Obéir à l'impératif catégorique signifie se positionner d'emblée ainsi.

Cette structure de l'autorité traverse toute la pensée kantienne, notamment dans deux figures essentielles, celle du savant philosophe autour de laquelle s'articule l'Université, et celle du législateur. C'est là au niveau institutionnel que l'impératif moral prend la forme d'une éthique de l'invisibilité comme légitimation morale du politique. Ainsi, au niveau de l'État, l'enjeu de Kant à partir de 1793 est de réussir le transfert politique de la conception morale de l'autorité. Bref, il s'agit de maintenir, au niveau de la formation de l'État, la structure du règne des fins, avec son législateur manquant. Il y réussit en accordant au chef de l'État des droits exceptionnels qui s'accompagnent du devoir de ne jamais revendiquer de propriété sur le pouvoir qu'il exerce. L'origine de son pouvoir est alors inaccessible au législateur. Il a donc le devoir d'être invisible comme volonté particulière (comme le libre arbitre, dans le vocabulaire kantien), et les sujets ont, quant à eux, le devoir de maintenir cette invisibilité en ne cherchant jamais, sous peine de mort, à connaître l'origine du pouvoir en place. Ce que j'appelle l'éthique de l'invisibilité est alors clairement identifiable dans la pensée kantienne, bien que sous d'autres noms: l'interdiction inconditionnelle de toute présentation déterminante du pouvoir, l'interdiction de toute résistance à l'État, à commencer par celle de tout questionnement sur la légitimité singulière de l'État en place. La structure de cette éthique fondatrice du politique est alors double: elle est donatrice de légitimité, c'est-à-dire élection de l'autorité et elle définit en même temps les conditions dans lesquelles cette autorité pourra s'exercer.

La figure du savant révèle, dès qu'elle est institutionnalisée, la même structure. Que le savant 
philosophe ne soit qu'un idéal n'a pas de quoi surprendre, la finitude ne donnant pas à l'homme accès à sa propre raison. Le philosophe dont parle la Critique de la raison pure est celui qui assigne des fins essentielles à l'usage de la raison et renvoie donc au savant du texte de 1784 sur les Lumières.

(...) il [le philosophe] ne se trouve lui-même nulle part, tandis que l'idée de sa législation se rencontre partout dans toute raison humaine (...) (262).

Le problème politique surgit lorsque cette figure du philosophe en vient à peupler l'Université. Apparaît alors un double-bind clairement énoncé par Derrida où le philosophe dans son retrait même [...] reste inévitable (1985: 24). Il faut des philosophes au sein de l'université, mais il ne faut pas qu'ils soient déterminables comme philosophes. Le philosophe de la Faculté de philosophie qui ne se légitime que de son retrait détermine alors la structure de l'université kantienne qui devra composer avec cette figure de l'invisibilité. La localisation du philosophe au sein de l'université se fait alors par un jugement qui discrimine selon une frontière invisible entre ceux qui sont bien là présents, les juristes, médecins et théologiens, mais qui ne participent pas à la détermination de la destination de l'université, et les philosophes qui eux fondent l'institution, lui assignent des fins, au prix de leur retrait, alors même qu'ils n'existent pas encore. L'université aujourd'hui, je le soutiens, est encore absolument kantienne, non pas dans sa destination, ou son organisation, mais dans le mode par laquelle elle assure l'institutionnalisation du savoir, c'est-à-dire l'intégration du savoir dans le juridique. Sur quel mode existe au présent une institution dont l'objet propre, le savoir, est toujours temporellement décalé, à venir comme projet, ou passé comme héritage? L'institutionnalisation s'appuie sur la légitimité de l'autorité comme devoir d'invisibilité, et c'est à ce prix que se maintient l'utopie égalitaire au sein du savoir. Une utopie dont la Modernité agonisante et postmoderne ne garde que la régulation d'une libre circulation du savoir. C'est à ce prix que s'institue pour Kant la communauté des savants, et c'est à ce prix que se monnayent aujourd'hui les savoirs. Un prix qui semblait bien léger jusqu'à ce que des corps tout autrement incarnés ne le révèlent comme une oppression politique. 


\section{La politique de l'invisibilité}

L'éthique de l'invisibilité détermine donc une politique dans la modernité kantienne, elle peut même se définir comme la fondation éthique du politique. Généralisant le concept, on peut définir aujourd'hui ce qu'elle détermine comme politique sous le nom de politique de l'invisibilité. La politique de l'invisibilité est une politique étatique (au sens où elle défend un territoire, et ce, qu'elle soit défendue par des hommes ou des femmes) motivée par le rêve d'une unité sociale qui serait assurée en imposant à tous le devoir de ne jamais faire obstacle à ce qui pourrait nuire à l'homogénéité du corps social. Il est demandé à chacun de se comporter dans l'espace public de telle sorte qu'il apparaisse toujours substituable à tous les autres. Il faut alors rendre invisible toute spécificité, tout ce qui nous identifierait comme Noir, Musulman, Gai, Juif, Anglophone, etc., tout ce qui donnerait l'illusion que l'on entretient des liens spécifiques avec certaines personnes plutôt que d'autres. Rendre invisibles les spécificités, cela signifie les retirer de l'espace public, les enfermer dans la vie privée ou dans des espaces strictement utilisés pour cette forme de socialité. Cette politique de l'invisibilité, c'est quelque chose comme une idéologie Schtroumpf. Dans le pays des Schtroumpfs, il y a le Schtroumpf paresseux, le Schtroumpf gourmand, le Schtroumpf musicien, et puis il y a la Schtroumpfette, et aujourd'hui, nous aimerons bien ajouter encore le Schtroumpf musulman, le Schtroumpf gai, le Schtroumpf noir, etc. Que la spécificité ne soit plus que des caractéristiques particulières, donc secondaires, qui n'affectent en rien la substituabilité des individus les uns aux autres. Ainsi s'effacent - espère-t-on - toutes les spécificités qui divisent le corps social. Si cette politique peut apparaître comme recherchant de bonne foi l'harmonie sociale, il ne faut pas se méprendre cependant sur ces effets réels.

La politique de l'invisibilité est une utopie dangereuse, elle définit une norme et un idéal à suivre dont le respect ne se paye pas au même prix si on fait partie de la majorité ou d'une minorité. En effet, être invisible, être quelconque, demande un effort considérable, car personne ne l'est. Etre blanc, hétérosexuel, est déjà en soi une spécificité que l'on partage avec certains mais pas avec d'autres. On n'est pas ici au pays des Schtroumpfs où il n'y a jamais qu'un Schtroumpf de chaque sorte. En fait, on n'est pas Femme, Gai, ou Noir seuls, on l'est toujours en relation avec d'autres. Et notre visibilité comme Femme, Gai, ou Musulman est toujours la visibilité des différences partagées, la 
singularité de chacun se marquant, quant à elle, à l'intérieur de ces différences. Le corps social n'est pas et n'a jamais été homogène, il est structuré par des différences collectives, qui sont souvent, - et de plus en plus, en raison des métissages de toutes sortes indéfinissables, mais qui marquent néanmoins un écart entre notre regard sur nous-mêmes et notre regard sur l'autre. Impossible alors de satisfaire pleinement l'éthique de l'invisibilité qui a comme effet pervers d'instaurer une hiérarchie de valeurs entre les individus. Plus vous vous approchez de l'idéal d'invisibilité, plus vous êtes considéré comme le représentant du corps social en entier, comme quelqu'un qui peut parler au nom de tous. Plus votre spécificité est visible, plus on cherchera à vous classifier, à savoir à quelle marginalité vous appartenez. Une hiérarchie que l'on reconnaît clairement à la demande de justification qui est imposée à ceux qui sont visibles. Si vous montrez une appartenance même indéterminée, si vous manifestez une différence même indéfinie, ce sera à vous de vous justifier, de rendre compte du pourquoi de ce que vous êtes: pourquoi portez-vous ce vêtement, parlez-vous avec cet accent, portez ce nom, pourquoi n'êtes-vous pas comme les autres? Que la question soit posée, dans la haine, la méfiance, ou dans la curiosité sympathique, il reste que le droit à la poser manifeste clairement que la justification appartient à l'autre. Or celui qui doit se justifier est celui qui a tort, qui a une dette à payer. La preuve en est que s'il refuse de donner l'explication de sa différence, de sa visibilité, il sera accusé d'être asocial, de rejeter les règles de la socialité. Et s'il ne veut pas et ne peut pas rendre compte de sa différence, il sera accusé d'être un imposteur, de renier ses origines, de se prendre pour ce qu'il n'est pas. Même la politique du multiculturalisme tombe dans le piège de l'éthique de l'invisibilité dans son désir de connaître, de catégoriser les différences en termes de cultures, d'origines, d'identités. Par contre, en vertu de cette même éthique, plus un membre social pourra passer pour invisible, moins il aura besoin de justifier son existence, il pourra entrer dans un lieu public, prendre la parole de droit, il n'aura pas pour être entendu à justifier d'abord pourquoi il est tel qu'il a l'air. Ainsi se trouve reconnu d'emblée un privilège à l'homme sans qualités (ohne Eigenschaften, qui n'a rien en propre, selon le titre de l'ouvrage de Musil), en toute position d'autorité.

L'éthique de l'invisibilité détermine ainsi une politique de la territorialité selon un mode tout à fait pervers. Elle inscrit des frontières invisibles entre ceux qui sont blancs (selon toutes les connotations de ce mot dans la langue française), et ceux qui ne le sont pas, et définit 
les conditions de la visibilité des corps, leur organisation et leur co-appartenance au sein de corps collectifs. Elle n'implique cependant aucune positivité dans la désignation de ceux qui seraient visibles, elle est plutôt une ligne de partage entre le privé et le public qui s'impose comme ségrégative lorsqu'un sujet refuse de laisser tomber du côté du privé une détermination, et de la rendre ainsi invisible, et plus généralement, chaque fois que le droit de parole est en question. Reconnaître cette territorialité, ramener au visible cette ségrégation que l'éthique de l'invisibilité maintient dans l'invisibilité est le premier geste politique de contestation de tout ce système. Le second est de comprendre que cette territorialité n'est que l'effet spécifique d'une politique de l'autorité dont l'acte fondateur est d'avoir constitué le projet d'une réglementation de la visibilité. Refuser alors l'éthique de l'invisibilité, c'est affirmer que la visibilité tout simplement ne se discute pas, ne se réglemente pas.

\section{Topographies de l'université}

L'université est le lieu privilégié de tout différend sur les politiques de l'autorité. Ainsi que les exemples du PC (Political correctness) le laissent entendre, elle est un des lieux institutionnels actuels où les conflits créés par l'éthique de l'invisibilité sont très clairement analysables. Pour le comprendre il faut considérer l'université pour ce qu'elle est, à savoir un lieu institutionnel, dans tous les sens du terme. Lieu spatial délimité par des frontières parfois floues (couloirs, terrains), parfois surdéterminées (salles de classe); lieu institutionnel, quadrillé par une juridiction qui porte aussi bien sur les comportements que sur l'évaluation des compétences, des enseignements, etc.; lieu symbolique où les individualités se retrouvent reliées, co-dépendantes dans un corps commun fracturé, divisé, mouvant, mais laissant toujours au moins une trace sur les corps singuliers. Une fois admise la condition postmoderne qui laisse l'université incapable d'assurer une unité dans une destination commune des différents corps de savoir qui la composent, il reste à comprendre ce qui assure à l'université sa spécificité comme lieu institutionnel, c'est-à-dire ce qui lie ensemble tous les membres qui circulent dans son espace. Cet espace est structuré aussi bien géographiquement que symboliquement par des sphères autonomes d'autorité qui relaient la légitimité universitaire. Ces sphères sont des départements, des classes, des centres de recherche, etc. Et c'est par rapport à elles que les membres du corps universitaire se définissent, se situent. Or elles 
sont désormais soumises à des conditions hétérogènes de légitimation, ce qui a permis précisément l'émergence d'Études spécifiques, féministes, gaies et lesbiennes, culturellement identitaires ... Il ne faut pas croire naïvement que ces sphères autonomes n'ont plus de compte à rendre à l'université dans son ensemble. Simplement, elle lie ensemble les multiples sphères d'autorité non pas dans une fin commune, mais dans l'interdiction de toutes fins spécifiques, donc par le droit qu'elle se donne de circuler à l'intérieur de toutes les sphères de savoir qui la constituent. Ce principe de circulation, de droit à l'accessibilité, se manifeste à travers la norme de l'excellence qui caractérise les universités post-historiques selon Bill Readings.

La crise postmoderne des institutions a produit une norme résiduelle qui est à l'idéal du savoir ce que la rationalité instrumentale est à la rationalité finale: l'excellence. A défaut de viser la constitution d'une communauté savante sous l'égide de la raison, ou la culture de l'humanité, ou la fondation de l'identité nationale, l'université actuelle recherche l'excellence. Mais il s'agit d'une norme indéterminée dont l'application ne peut être que circulaire. Elle s'applique en effet à l'intérieur d'une rivalité entre les universités au sein d'une joute internationale dont l'enjeu est tout à la fois l'excellence et la définition des critères de l'excellence. Appliquons donc un tel critère à l'institution d'Études gaies et lesbiennes. Une université promouvra de telles Etudes si celles-ci sont un signe de son excellence. Le problème est qu'elles ne seront des signes d'excellence que si elles sont déjà légitimées et institutionnalisées comme marques d'excellence au niveau du jeu international. La règle de l'excellence est en ce sens une règle perverse puisqu'elle n'est applicable qu'à ce qui existe déjà et non à une revendication de reconnaissance. De façon générale, l'exigence imposée très souvent aux Études gaies et lesbiennes de justifier leur spécificité avant leur institutionnalisation est perverse dans la mesure où l'établissement de cette spécificité est précisément ce qui est visé par ces Études et ne peut être qu'un résultat non un principe fondateur. C'est donc dire qu'à la différence des destinations passées de l'université, le principe d'excellence est un principe purement intrauniversitaire, ce qui explique son caractère tautologique. Il ne sanctionne que ce qui est déjà fondé.

Cependant, il faut comprendre que la juridiction universitaire (dont la norme de l'excellence fait partie) n'est qu'une des facettes de l'université. Elle correspond 
au mode selon lequel l'université gère les échanges en son sein. Sur ce point précis, l'université actuelle reste assez près de ce que Kant décrit comme condition de l'institutionnalisation dans son écrit Le conflit des Facultés. Pour Kant, la fondation de l'université (c'est-àdire à la fois sa légitimité et sa spécificité) repose sur la Faculté de philosophie qui, elle-même, s'oriente selon des fins purement rationnelles. Mais ceci dit, l'institutionnalisation de l'Université est l'affaire de l'État qui en définit les limites et la juridiction. Cette dissociation entre le mode juridique d'organisation et le but du savoir lui-même survit aujourd'hui même si l'Université s'est détachée partiellement de l'État. Le principe de l'excellence quadrille donc l'espace institutionnel de l'Université. Mais il ne peut remplir sa fonction - donner sa spécificité et sa cohésion - que parce qu'il est l'émergence d'un principe fondateur qui tisse entre les membres du corps universitaire des liens symboliques d'appartenance à un lieu commun. En effet, ne sanctionnant que ce qui est déjà fondé, il présuppose un tel geste fondateur. Ce que Derrida conclut de son analyse de Kant est encore tout à fait pertinent : la fondation d'un droit n'est pas plus légitime ou juridique que la fondation d'une Université n'est un événement universitaire, intra-universitaire (Du droit à la philosophie, 334).

Derrière le principe d'excellence se cache le droit que l'université se donne d'avoir accès aux différentes sphères autonomes de l'université. Il fournit non seulement une mesure commune, mais définit l'accès aux savoirs spécialisés en termes quantitatifs. On sait que la surspécialisation des disciplines universitaires rend difficile de les juger de l'extérieur, seuls des spécialistes peuvent juger de la valeur d'un spécialiste. Mais avec le critère d'excellence, l'inaccessibilité de fait est compensée par une accessibilité de droit. En effet, si l'accessibilité n'est qu'une question de quantité de connaissances ou d'activité de recherche, elle est hypothétiquement donnée à qui se donne la peine de remplir ces prérequis. Ainsi, l'université de donne bien le droit et les moyens de circuler librement à travers les différentes sphères. Dès lors, dans ce système, la pluralité des disciplines, la création de disciplines nouvelles devient tout à fait autorisée du moment que chacune laisse à l'institution un droit d'accès (même si ce droit est en fait souvent inapplicable). L'hétérogénéité des discours de légitimation (qui caractérise la condition postmoderne) est parfaitement admissible dans le cadre de ce système de circulation qui maintient une forme minimale d'incorporation institutionnelle. Certes, dans les faits, les nouveaux discours auront à rencontrer les 
obstacles et les résistances crées par les discours traditionnels tenus par les différentes sphères. Mais ces conflits resteront internes, l'université sanctionnant le vainqueur.

On peut reconnaître maintenant dans le principe de la circulation une application de l'éthique de l'invisibilité. Il s'agit bien d'une forme d'incorporation négative, on crée une unité, non par la formation d'une totalité, mais simplement négativement en interdisant toute frontière interne qui s'opposerait à la constitution d'une totalité si une telle chose était possible. De la même façon, le devoir de n'être rien est la forme légitime de l'autorité dans la mesure où il est la condition idéale de la circulation. Du coup, le véritable enjeu des Études féminines, des Études gaies et lesbiennes, des Études afro-américaines, ou des Études islamiques, n'est pas seulement la légitimité d'une discipline nouvelle, mais l'existence même d'un tel corps au sein de l'université. La visibilité et l'existence sont une seule et même chose. Pour un gai, une femme, une lesbienne, un Noir, un Musulman, ne pas avoir le droit à la visibilité est une seule et même chose que de ne pas exister comme corps collectif dans l'enceinte de l'Université. Soumettre les corps collectifs à la contingence d'une revendication de légitimité, c'est déjà créer un différend et une victimisation possible. Les corps spécifiques n'ont pas à justifier leur existence, ils existent. C'est l'éthique de l'invisibilité qui les force à donner raison de leur existence en leur donnant a priori tort de ne pas disperser leurs particularités dans les différentes vies privées. La légitimation des formes institutionnelles (programme, cours, etc.) que prendra cette présence visible ne peut être qu'un moyen d'avoir accès à la visibilité et non les conditions de la visibilité. Ce renversement de paradigmes, de la légitimation à la visibilité, entre en conflit direct avec le principe de circulation qui fonde l'Université actuelle. La visibilité signifie en effet l'opacité.

Ce qui a lieu dans une sphère universitaire (un cours, un programme, un département, etc.), ce n'est pas seulement la transmission d'un savoir quantifiable, c'est surtout la création d'une certaine topographie structurant les corps collectifs. Un cours d'Études gaies, par exemple, ne met pas seulement en jeu une certain contenu de savoir, il oblige les membres qui partagent un même espace, l'enseignant, les étudiants, les conférenciers invités, s'il y en a, à se définir les uns par rapport aux autres en fonction de la visibilité ainsi produite. Je ne veux absolument pas dire que les participants, professeur compris, devront dire s'ils sont 
gais ou non. La visibilité d'un corps collectif rend caduque ce type d'identification. Par opposition, lorsque la visibilité n'est pas donnée, celui qui choisira de travailler sur les Études gaies devra porter seul le poids politique de son choix, et mettre son identité en jeu dans ce geste. Rencontrer un directeur de thèse et lui proposer de diriger une thèse sur les Études gaies équivaut généralement à un Outing. Un cours d'Études gaies par contre constitue une situation sensiblement différente. Il est fort probable que de tels cours sont fréquentés surtout par des Gais. Mais là n'est pas la question. Car le cours rend visible non une identité gaie singulière, mais un désir pour le corps gai comme corps collectif. Et montrer, ce n'est pas dire. Libre au tiers voyeur de se demander si un tel qui assiste à ce cours est gai ou non. Mais s'il doit se poser la question, s'il est soumis à l'incertitude de sa déduction, c'est que le droit de parole ne lui est pas donné d'emblée. Il faut reconnaître alors que la différence entre le corps gai et l'individu est maintenu. Reste ouverte en même temps la possibilité de participer à ce désir commun pour ceux qui ne se considèrent pas gais. De même, n'est plus pertinente la question de savoir si le professeur doit être certifié gai pour enseigner un tel cours, car il aura de toute façon la charge de justifier sa présence en regard d'un corps commun désormais visible. Et ici on voit donc comment l'affirmation de la visibilité renverse l'éthique de l'invisibilité où la légitimité d'une autorité dépend de son haut degré de substituabilité. Ce qui est dit ici des Études gaies peut tout à fait s'appliquer aux Études féministes qui offrent aux femmes (et par extension à tous) la possibilité de manifester leur désir pour le corps collectif féminin autrement qu'en ayant l' air d'une femme.

Selon l'éthique de l'invisibilité, les fonctions de médiation (les positions d'autorité) sous lesquelles une pluralité se regroupe sont assurées par ceux qui présentent le plus haut degré de substituabilité. De même à l'université, ces fonctions (qu'il s'agisse d'une individualité administrative ou du corps singulier du professeur) sont associées à une légitimité définie par la substituabilité, c'est-à-dire en relation à un savoir quantifiable, accessible de droit à tous ceux qui veulent l'acquérir et qui, n'étant incarné d'aucune façon par son porteur, est une marque d'invisibilité. Ces médiations ne sont légitimes alors qu'à titre de rouages de transmission et sont assurées au mieux, selon la logique du système, par ceux qui présentent un haut degré d'invisibilité. Toute personne ne répondant pas à ces critères se trouve sommée de justifier sa particularité, ce qui au pire la rend inadéquate à sa fonction (peut-on 
demander à un gai d'enseigner la littérature française du XVIIe?), au mieux condamnée à représenter une forme limitée de savoir, celle correspondant à son groupe (aux gais les Études gaies, aux femmes les Études féministes...). Mais la nécessité de la justification est déjà un signe de faiblesse. Au contraire, en constituant des corps collectifs, on crée des zones d'opacité et de résistances à la circulation et à la substituabilité. En effet, la constitution d'un corps collectif signifie l'introduction d'une différence dans le corps social, une différence par rapport à laquelle chacun devra se définir. $\mathrm{Au}$ sein de l'université donc, que l'on soit le médiateur institutionnel (en l'occurrence, le professeur), ou l'étudiant, il faudra toujours se définir par rapport à cette différence qu'institue la visibilité d'un corps spécifique. Outre le déplacement de la position professorale qui n'est plus le point de substituabilité dans un public homogène, il en résulte une opacité du point de vue de l'institution dans son ensemble. Non pas qu'il se transmet en ce lieu un savoir d'initiés, mais les effets du discours ne sont repérables que par rapport à la topographie et dépendent donc de la position que l'on occupe au sein de la topographie. En effet, l'impact qu'aura un cours d'Études gaies ne sera pas le même si l'auditeur s'incorpore au corps gai ou non. Les analyses féministes se sont déjà penchées sur ce type de question, sur l'impact qu'a dans la transmission du savoir le sexe des intervenants (Jane Gallop). De telles analyses mériteraient d'être généralisées, et je suggérerais ici l'usage de la théorie psychanalytique, bien que non exclusivement. Et toutes ces variations dans les effets du discours échappent au contrôle de l'institution. Il ne devient plus possible de circuler librement dans les sphères autonomes, car la circulation présuppose un agent neutre, indéterminé, pouvant s'intégrer à tous les corps spécifiques, et passer grâce à son invisibilité pour un membre de ces corps, passer pour Noir, Femme, Gai, Musulman, etc.

Il faut conclure sur quelques remarques de prudence. Il est clair qu'à l'heure actuelle, il n'est pas bon de contester l'éthique de l'invisibilité comme détermination du lieu de l'autorité. En effet, redonner une présence, une détermination propre à l'autorité, $\mathrm{c}$ 'est rien de moins que le totalitarisme. Une autorité qui ne se légitimerait d'aucun retrait serait nécessairement totalitaire, puisqu'elle prétendrait coïncider avec le groupe qu'elle recouvre. La question est plutôt de reconnaître que l'éthique de l'invisibilité détermine des exclusions et une subtile et invisible ségrégation. Ce faisant, on révèle un nouvel enjeu politique, objet de différends (au sens où Lyotard entend ce terme) : le corps dans sa visibilité. Cet 
objet-enjeu subit des formes de contrôle et même d'oppression très spécifiques de la part des politiques qui se légitiment de l'éthique de l'invisibilité. La conscience nouvelle que je réclame touche aussi l'avenir des Études spécifiques que je défends ici, dans la mesure où la politique de l'invisibilité peut tout à fait les atteindre, si elles oublient que leur finalité première n'est pas la constitution d'une territorialité propre, mais la modification dans la circulation du savoir, la formation du corps collectif et l'invention de formes nouvelles d'autorité qui refusent la logique de l'éthique de l'invisibilité, y compris lorsque celle-ci impose le devoir de s'identifier.

\section{BIBLIOGRAPHIE}

Derrida, Jacques, Du droit à la philosophie. Paris:

Galilée, 1990.

Derrida, J., Force de loi. Le fondement mystique de l'autorité . Paris: Galilée, 1994.

Joos, Jean-Ernest, Kant et la question de l'autorité. Paris: L'Harmattan, coll. La philosophie en commun , 1995.

Kant, Immanuel, 1781, 1787: Critique de la raison pure, trad. A. Tremesaygues et B. Pacaud. Paris: P.U.F., 8e éd., 1975.

1784: Réponse à la question : qu'est-ce que les Lumières? trad. S. Piobetta, in La philosophie de l'histoire. Paris: Montaigne/Gonthier, 1947.

1785: Fondements de la métaphysique des moeurs, trad. V. Delbos. Paris: Delagrave, 1977.

1793: Sur l'expression courante : il se peut que ce soit juste en théorie, mais en pratique cela ne vaut rien, Deuxième section, trad. Louis Guillermit. Paris: Vrin, 1967.

Readings, Bill, The University in Ruins. Harvard University Press, 1996. 